\title{
Reforming ICT \\ Graduate Programs \\ to Meet Professional \\ Needs
}

Sergio Luis Toral, María Rocío Martínez-Torres, and Federico Barrero

University of Seville

\section{A cross-analysis of EU masters' programs and professional profiles in information and communication technology reveals deficiencies in many areas. Reform initiatives are already addressing some of these deficiencies, but work remains.}

nformation and communication technology (ICT) has almost unlimited application in the current knowledge era, and the onus is on higher education institutions to nurture that potential. Unfortunately, ICT curricula design does not always support the competencies and professional profiles that society and the ICT industry demand. In recognition of this gap, many EU nations are attempting to reform their higher education systems. In part, various national developments trigger reforms, but there is also the desire to share in quality gains to maintain and raise the standards of higher education systems across Europe. The Bologna Process, ${ }^{1}$ for example, focuses on legal reforms as well as the evolution of a pedagogical framework that seeks to involve students more in the teaching process.

As "The Bologna Process" sidebar describes, one of the main goals is to establish the European Higher Education Area (EHEA), which aims to promote learner-centered approaches, moving the focus from teacher to student. ${ }^{2}$ In light of this shift, curriculum designers must seek to understand relevant competencies - the knowledge, skills, and attitudes that students must exhibit to transition to professionals in a particular field. These competencies must also promote lifelong learning and reinforce cross-training.

The EU has launched several initiatives to define such competencies, notably the Tuning Project, ${ }^{3}$ Definition and Selection of Competencies (DeSeCo), ${ }^{4}$ and Career-Space
(www.eaeeie.org/theiere/meeting_norway/Career-Space PanICT_GENIUS.ppt). Of these, Career-Space is the most relevant to ICT and carries recommendations both from the EU government and from key players in the ICT industry. Work to date has produced in-depth professional profiles in ICT.

To aid in the work of better understanding the competencies associated with these profiles, we examined ICT master curricula from universities in countries actively involved in the Bologna Process and compared these programs with the profiles from the Career-Space initiative. Our results show that only 8.6 percent of these programs cover two or more profiles, revealing profound weaknesses in ICT management. These areas could serve as a kind of roadmap for curriculum design reform, since the aim of reformation projects is to bring their curriculum in line with what practitioners want.

\section{PROJECTS TO DEFINE COMPETENCIES}

The global movement to competency-based training has naturally put the focus on defining "competence," which at present has a range of meanings and interpretations, depending on discipline and context. Roughly, competence is the specialized system of abilities, proficiencies, or skills needed to reach a specific goal. ${ }^{5}$ An important implied element of this definition is the relation between competence and performance, which links competence to social 
action, such as performing the skills in social situations. ${ }^{5}$ Consequently, competence is not limited to skill acquisition, but encompasses the ability to handle challenges that occur in a specific environment. Indeed, competencies are expressed and performed through actions, which are generally related to a specific social context. ${ }^{6}$

Figure 1 shows the steps to acquire a competency. The process begins with information acquisition within a network of meaning, which leads to knowledge. Applying that knowledge to a specific context can lead to ability. Ability in turn must be combined with a specific attitude, including values and motivation, to result in some act to demonstrate that ability. If the action is consistent with a set of adequacy or appropriateness standards, it leads to competence. In the final step, competence combines with responsibility to complete the transition to professionalism. ${ }^{6}$

European nations have launched several projects to define new competencies and more deeply understand existing ones. The Tuning Project, DeSeCo, and CareerSpace have all resulted from a movement to define and establish competencies as part of the larger aim to reform higher education in the EU. ${ }^{3}$

\section{The Tuning Project}

Coordinated by the University of Deusto in Spain and the University of Groningen in The Netherlands and supported by the European Commission, the Tuning Project aims to study the educational structures and content in higher education programs. ${ }^{3}$ To date, 100 European universities are participating in the effort to understand both discipline-specific and generic competencies. The project's guiding notion is that reforms in higher education lead to the tuning of curricula, particularly the teaching of competencies. ${ }^{3}$ The academic and professional profiles required by society are important in this process, but so is the need to express education level in terms of competencies and learning outcomes.

\section{Definition and Selection of Competencies}

DeSeCo emphasizes the significance of general competencies at the global level, ${ }^{4}$ classifying key competencies in three broad categories within a conceptual framework.

The first category concerns tool competency. Individuals must be able to use a wide range of tools to interact effectively with the environment-both the physical surroundings, such as the technology, and the social-cultural context, such as the use of a particular language. In other words, users need to understand these tools well enough to adapt them for their own unique purposes, and to use them interactively.

The second competency framework category is the ability to interact in heterogeneous groups - a skill vital to an increasingly interdependent world of individuals with diverse backgrounds.

\section{$\rightarrow$ THE BOLOGNA PROCESS}

he Bologna Process is a European reform project aimed at establishing a European Higher Education Area (EHEA) by 2010. One of the guiding principles is to change the role of students from passive actors to active learners, which makes this more than just a legal reform; it is also a motivating force in changing the pedagogical framework. The process, launched by the European Commission, is driven by 46 countries that have adopted new higher education legislation to introduce and regulate elements of the Bologna Process, although countries vary in how quickly they are implementing these elements, depending on national agendas and on different experiences and traditions regarding higher education policy making.

All higher education systems use the European Credit Transfer and Accumulation System with the goal of achieving a fully transparent higher education area. As a result, many learners now have the option to continue second- or third-cycle studies in other institutions in the same country or in other EHEA countries. Additionally, mobility from other parts of the world toward the EHEA has increased substantially.

Quality assurance is another guiding principle of the Bologna Process, with the intent to develop comparable criteria and methodologies. The process has adopted the European Standards and Guidelines in concert with the European Quality Assurance Register for Higher Education. This joint adoption represents a significant achievement in international cooperation because it is the first mechanism in Europe aiming to identify quality assurance agencies that operate within the EHEA.

The third competency category concerns an individual's ability to take responsibility for managing his or her own life, to situate that life in the broader social context, and to act autonomously.

\section{Career-Space}

Career-Space is the only initiative that considers the specific competencies for ICT professionals. The CareerSpace Consortium (CSC), which consists of 11 major European ICT companies-BT, Cisco Systems, IBM Europe, Intel, Microsoft Europe, Nokia, Nortel Networks, Philips Semiconductors, Siemens AG, Telefónica, and Thaleshas the support of the European Commission and the European Information, Communications and Consumer

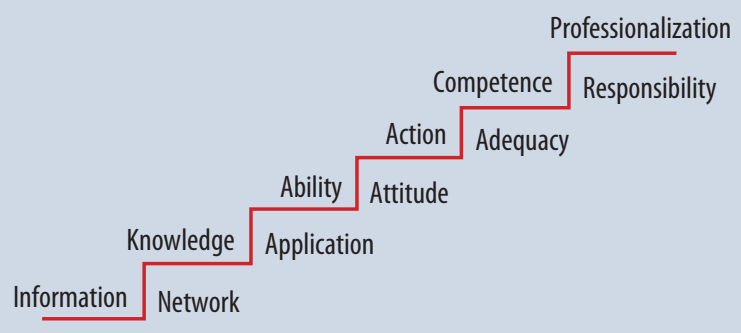

Figure 1. Steps toward professional development. In addition to skills acquisition, competency requires certain attitudes and actions to demonstrate those skills in a social situation. 


\section{$\rightarrow$ CAREER-SPACE CONSORTIUM RECOMMENDATIONS}

$\mathrm{n}$ the current knowledge economy, the ICT sector has become a popular focal point of higher education because of its expected economic benefits.' At present ICT has five key characteristics:

- highly dynamic technological change with rapid penetration and adoption rates;

- decreasing costs for new equipment and features;

- rapidly increasing range of applications and penetration in many realms of professional and personal life;

- an intertwined institutional marketplace, with the private sector acting in a decreasingly regulated environment; and

- dependence on a range of qualities of skilled human resources.

These characteristics suggest the need to focus attention not only on the quantitative availability of ICT practitioners, but also on the skills required for graduates to be more work-ready for the tasks and problems encountered in complex ICT business and work processes.

In light of these characteristics, the Career-Space Consortium ${ }^{2}$ has made several recommendations to address ICT industry needs. ${ }^{3}$

\section{Create new ICT programs}

New programs should strive to give students a combined qualification from both the engineering and informatics cultures, as well as from related disciplines, such as business and behavioral skills.

\section{Combine electrical engineering and informatics elements}

Although traditional engineering and informatics programs are still needed, CSC recommends the creation and development of new curricula containing elements from electrical engineering, elements from informatics, and a significant focus on the teaching, training, and practice of behavioral and business skills.

\section{Take a systems view}

A broader systems view and the corresponding ability to create complete system solutions seem to be seriously lacking in many graduates entering the ICT industry.

\section{Require business knowledge}

A clear understanding of the fundamentals of business is vital to well-rounded ICT graduate training.

\section{Mandate new behavioral skills}

Specialists now work as part of multidisciplinary development teams, which are often international and geographically distant. The trend for products to be designed for a global market is growing, and national boundaries are becoming less relevant. Marketing staff and customers tend to be involved in the development of a product right from the start. An ICT graduate needs to be able to work with people from different cultures and backgrounds to arrive at a mutual understanding. Any university curricula should focus on these behavioral aspects.

\section{Increase mobility between academia and industry}

In addition to encouraging student mobility, mobility of university lecturers and professors and ICT industry representatives should be encouraged.

\section{References}

1. W. Petersen and C. Wehmeyer, "European Qualification Strategies in Information and Communications Technology (ICTTowards a European ICT Skills and Qualification Framework," Studies in Informatics and Control, vol. 14, no. 1, 2005, pp. 37-46.

2. Career-Space Project, Generic ICT Skills Profiles, Office for Official Publications of the European Communities, 2001.

3. Career-Space Project, Curriculum Development Guidelines: New ICT Curricula for the 21st Century: Designing Tomorrow's Education, Office for Official Publications of the European Communities, 2001.
Electronics Industry, and is coordinated by International Cooperation Europe Ltd.

Among the CSC's most recent efforts is work to determine a clear framework for students, educational institutions, and governments that will describe the roles, skills, and competencies required by the European ICT industry. The "Career-Space Consortium Recommendations" sidebar summarizes its guidelines. Basically, according to the CSC, ICT graduates should have a solid foundation in technical skills from both the engineering and informatics cultures, with a particular emphasis on

Table 1. Allocation of core skills for an ICT masters' program.

\begin{tabular}{|c|c|}
\hline Skill area & Percentage \\
\hline Scientific base & 30 \\
\hline Technical base & 30 \\
\hline Applications and systems thinking & 25 \\
\hline Personal and business skills & 15 \\
\hline
\end{tabular}

a broad systems perspective. They also need training in teamwork, with experience on real team projects, as well as a basic understanding of economics, market, and business issues. Finally, they need the personal skills essential to ICT practitioners: problem solving, communication and persuasion, lifelong learning, readiness to understand the needs of their customers and colleagues, and awareness of cultural differences when acting in a global environment.

To achieve these skills, the CSC recommends that core qualifications during ICT education should reflect the breakdown in Table 1. Most programs miss the last two rows of the table, which acknowledge the need for application knowledge in accordance with workplace demands for a particular job profile.

The CSC recommends that designers of electrical engineering curricula consider this framework as a way to achieve the employability that is a key goal of the European Higher Education Area. ${ }^{2}$ The CSC's intent is to further educational innovation that will satisfy near-term ICT requirements. As such, the recommendations should not 
be viewed as a prescription for curriculum design, but rather as information about ICT industry needs and guidelines that a university might use to increase its program's coverage of professional competencies.

\section{DERIVING PROFESSIONAL COMPETENCIES}

According to the CSC, ICT graduates need a solid background of technical competencies in both engineering and informatics, as well as a wide systemic perspective. Students need to reinforce teamwork competencies and experience a real project, in which several activities are developed in parallel. Learners should also have a basic knowledge of the economy, markets, and companies. ICT graduates will need interpersonal skills, the ability to solve problems and seek learning opportunities, the acuity to determine customer and colleague needs, and a consciousness of the multicultural context. The CSC's recommendations reflect those in related initiatives, such as DeSeCo.

On the basis of these findings, the CSC developed the 18 ICT professional profiles listed in the "ICT Professional Profiles" sidebar. The profiles represent the main areas related to both the ICT sector and other sectors that ICT crosses, such as management. Competencies derived from disciplines such as economics, business, and social sciences are becoming even more important than technical skills.

Each of the 18 profiles also describes a set of behavioral and technical skills (shown for the data communication engineering profile as an example) and includes a job description and set of tasks related to each job.

\section{CURRICULUM-TO-PROFILE ANALYSIS}

Using the provided information for each profile, we crossanalyzed the current offerings of ICT masters' programs in the EU with aspects of the professional profiles that these programs aim to cover. The programs are from universities in 13 European countries: England, Finland, France, Germany, Greece, Italy, Norway, Poland, Portugal, Scotland, Sweden, Switzerland, and The Netherlands. We reviewed 81 ICT masters' programs, extracting curricula from the university websites. We selected only programs officially recognized by the education ministry in that nation.

\section{Compliance per profile}

To determine the number of CSC profiles covered, we first calculated the average level of compliance for an individual profile, which entailed a deep study of each master's program. Because it was difficult to assign a numerical value to compliance, we settled on high, medium, and low compliance ranges. To rate a high compliance, the curriculum had to cover 80 percent or more of the profile description and tasks; to rate a medium value, the curriculum had to cover between 40 and 80 percent; and a curriculum with a low value had less than 40 percent coverage.

\section{$\rightarrow \quad$ ICT PROFESSIONAL PROFILES}

ur cross-analysis compared masters' degree programs in ICT to 18 professional profiles specified by the Career-Space Consortium. Each profile also specifies a set of behavioral and technical skills. The data communications engineering profile is expanded as an example:

- Communications network design

- Data communications engineering

- Behavioral skills: analysis, creativity, teamwork, communication, professional attitude, problem solving, initiative, risk management, flexibility and self-learning, efficiency and quality, commitment to excellence, and customer orientation.

- Technical skills: knowledge of protocols, requirements analysis, system architecture, software architecture, computer programming, troubleshooting of technical problems, objectoriented analysis and design, system integration, work estimation and scheduling, understanding and evaluation of internal and external specifications, and electronics theory and know-how (analog/digital).

- Digital design

- Digital-signal-processing applications design

- ICT management

- ICT marketing management

- ICT project management

- ICT sales management

- Integration and test/Implementation and test engineering

- IT business consultancy

- Multimedia design

- Product design

- Radio-frequency engineering

- Research and technology development

- Software and applications development

- Software architecture and design

- Systems specialist

- Technical support

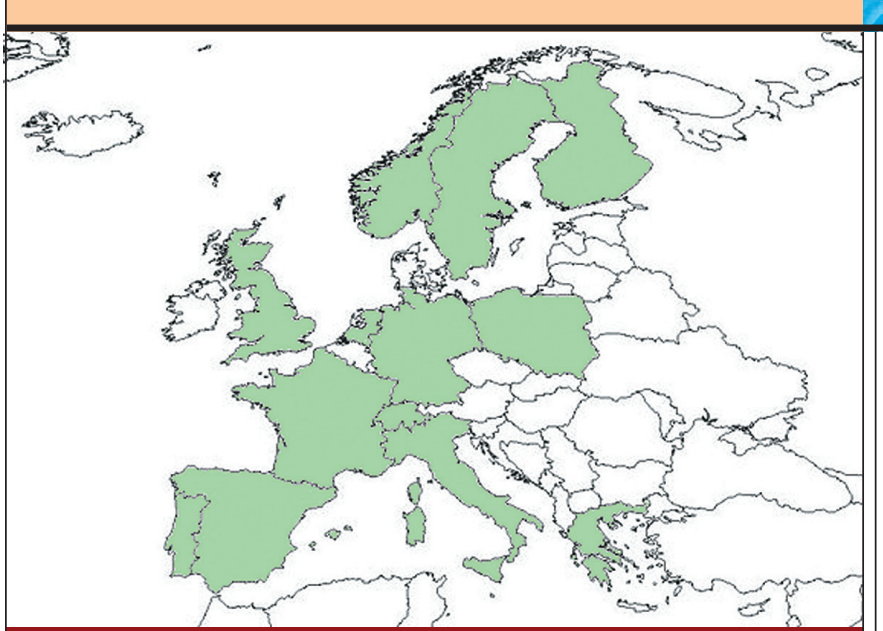

Figure 2. How well EU masters' programs comply with a particular CSC profile. Compliance is ranked high, medium, or low. In general, software-oriented ICT masters' programs comply with more profiles than hardware-oriented programs.

Figure 2 shows the results of profile compliance. Software architecture and design, multimedia design, and 


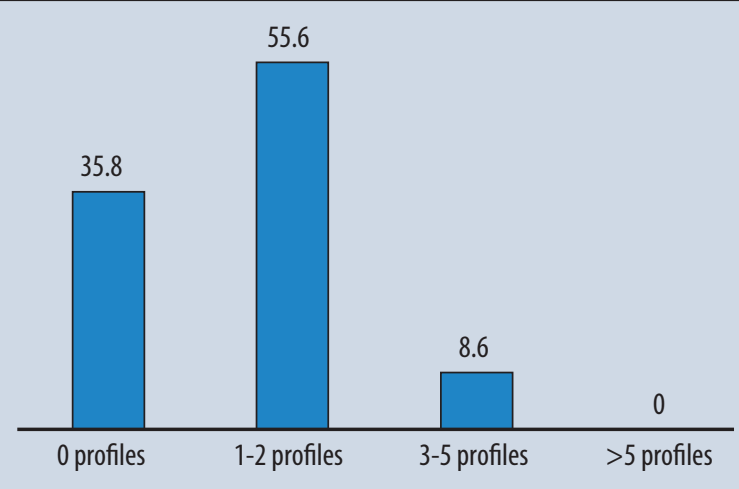

Figure 3. How well EU masters' programs cover the CSC's 18 ICT professional profiles. A profile is considered covered if the program complies with 80 percent or more of the profile skills. Most ICT masters' programs cover at most two profiles, and almost one-third do not cover any profile. Many concentrate on technical profiles almost to the exclusion of the management elements.

systems specialist are the most well-covered profiles; radio frequency engineering, product design, project management, and ICT management are the worst-covered ones. In general, software-oriented ICT masters' programs are more prevalent than hardware-oriented programs and comply with more profiles.

According to the CSC's curriculum development guidelines, personal and business skills should represent around 15 percent of an ICT curriculum. Sixty-nine percent of the ICT masters' programs we analyzed exceed that threshold.

\section{Profile coverage}

Once we determined individual compliance for a program, we looked at how many of the CSC profiles it covered (meaning that it had a high compliance for that profile). As Figure 3 shows, 55.6 percent of the ICT masters' programs cover at most two profiles, 35.8 percent do not cover any profile, and 8.6 percent address more than two profiles.

We found that the programs suffer from two major flaws. The first is poor professional orientation; the programs are simply not planting and growing the skills that ICT companies demand. The second weakness is that programs try to cover too much in too little depth and thus rarely reach an adequate compliance level. Most programs concentrate on technical profiles, with only 10 percent attempting to cover elements of the management profiles.

\section{IMPLICATIONS FOR CURRICULUM DESIGN}

Curriculum design, like education as a whole, relies firmly on the explanation of theoretical phenomena, but its practice cannot be considered theoretical. Rather, curriculum design is an applied science, ${ }^{7}$ so a conceptual framework and general guidelines are more relevant than a curriculum design theory. Even so, several authors have advocated a more systematic curriculum design approach ${ }^{7}$ that has two important features. The first is the idea that curriculum should take a student through a competency continuum. Thus, curriculum design must begin with a clear definition of what competencies the learner is expected to achieve. The learner's performance moves along the continuum starting at the point that fits his current performance.

\section{Design perspectives}

Traditionally, designers have followed either a bottomup or top-down curriculum design perspective. ${ }^{7}$ In the bottom-up view, scientific tradition and the course providers' special interests determine course content. The final curriculum is the result of aggregating courses in a somewhat structured set after negotiations among course providers. The quality within a course tends to outweigh the need to interface and coordinate with other courses.

In the top-down view, the course's intended contribution to the curriculum's educational objective determines course content. Each course must resolve the tensions between its intended contribution versus scientific tradition, disciplinary boundaries, and the course providers' specific interests.

\section{The case for reform}

Current educational reforms are aligned with the topdown perspective, with interests shifting toward the skills and competencies students must achieve to reach global educational objectives. The learner-centered practices promoted by the EHEA have increased attention to the learning performance. However, the setting of the global objectives should also reflect the pursuit of professionalism. It is alarming that so few ICT masters' programs cover CSC professional profiles in any depth or that they elect to shallowly cover many profiles. Neither of these approaches is consistent with the EHEA objectives. Clearly, global educational objectives are not in line with the addressed professional profiles.

Three universities have already taken steps to realign their programs, and their efforts should serve as reference points for those involved in curriculum design. In each case, the universities recognized that skills and competencies definitions are useless if they are not oriented to the specific professional profiles required by industry and society.

University of Lancaster. The university decided to enhance its engineering degree courses by adding a course on engineering applications so as to better prepare students for engineering careers. ${ }^{8}$ After concluding that they should derive course objectives from studies of actual engineering work, course designers created a taxonomy of professional educational objectives to bridge job descriptions and course objectives. They also stress the need to plan courses in terms of learning experiences. 
Delft University of Technology. The university has proposed a top-down approach to designing its systems engineering, policy analysis, and management curricula. ${ }^{7}$ From a graduate profile definition, designers derive knowledge clusters, components, and elements. This process reflects the classic design practice of reducing complexity through decomposition-an approach that requires specifying relationships among subsystems. Each knowledge cluster comprises several components that are explicitly linked to engineering skills. Components, in turn, comprise knowledge elements, each of which represents a theory or skill that the student must master to rate a certain competence level.

The main advantage of this systemic approach is its rationality. Explicitly formulating objectives, relationships, and mechanisms for evaluation and improvement creates a transparent development process that facilitates system optimization. The main drawbacks are the risk of failure in goal formulation, design complexity, and a lack of commitment from course providers, who might perceive that the process is taking away their creative freedom.

University of Seville. The university has proposed a general curriculum design methodology based on concept mapping, ${ }^{2}$ which is based on ranked attributes from a set of target professional profiles.

Figure 4 shows the rated cluster map that the university used as the starting point for designing its electronic engineering (EE) degree curriculum. The first step in concept mapping is to form a working group of academic staff, practitioners, students, and practitioners in relevant fields, who then review professional profiles and select attributes that could form the basis for a curriculum. The EE working group, for example, reviewed the digital design, digital signal processing, applications design, and product design CSC profiles, choosing 98 attributes (blue points in the figure). Group members then individually classify the attributes under some topic using their own criteria and rate their value on a scale of 1 to 7 , with 7 having the most value.

To generate the cluster map, an analyst performs multidimensional scaling and a cluster analysis on these selections. The working group as a whole determines the final number of clusters, trying to keep each one homogenous (no multiple meanings). Generally, the more layers a cluster has, the more important it is and the higher its priority in the final curriculum. Table 2 lists the clusters the EE group decided on, which correspond to the numbered shapes in Figure 4.

Each cluster's rating depends on the individual rating of items in that group. The analyst decides how many clusters a map will have, checking that the items included in each cluster have a common meaning. As a rule, too many clusters are better than two few because there is less risk that a single cluster will contain heterogeneous concepts. Even

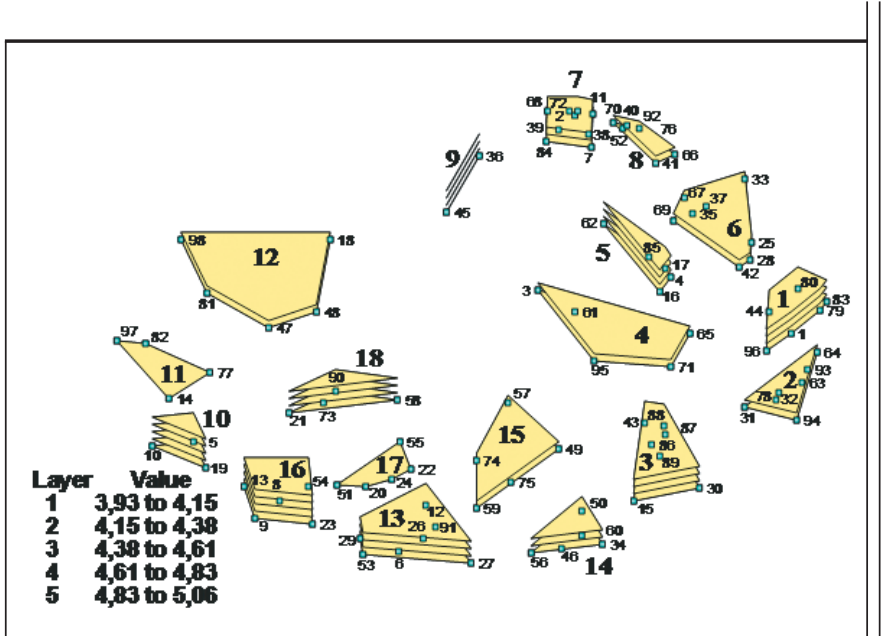

Figure 4. A concept map for designing the EE degree curriculum at the University of Seville. A curriculum design working group creates the map from a set of attributes in related professional profiles. In this case, the group has identified 98 attributes (blue points). Each member then separately classifies and rates each attribute on a scale from 1 to 7 , which represents the attribute's value range in a particular cluster (bottom left). Finally, analysts use multidimensional scaling and cluster analysis to obtain the map. The resulting layered clusters (numbered shapes) correspond to topics the curriculum must address.

Table 2. Clusters in the concept map for designing an EE degree curriculum.

\begin{tabular}{|c|l|}
\hline Cluster & \multicolumn{1}{c|}{ Description } \\
\hline 1 & Production procedures \\
\hline 2 & Fault diagnosis/fault solution \\
\hline 3 & Manufacturing technologies and design \\
\hline 4 & Industrial processes for electronic design \\
\hline 5 & Legal regulation and standards \\
\hline 6 & Electronic project management \\
\hline 7 & Personal and instrumental competencies \\
\hline 8 & Systemic competencies \\
\hline 9 & Other competencies \\
\hline 10 & Instrumentation \\
\hline 11 & Programming knowledge \\
\hline 12 & Informatics applied to electronics \\
\hline 13 & Basic electronics \\
\hline 14 & Power electronics \\
\hline 15 & Emerging technologies \\
\hline 16 & Sensors and signal conditioning \\
\hline 17 & Microprocessor systems and applications \\
\hline
\end{tabular}

small clusters are retained because each cluster reflects an aim of the target professional profiles, however small.

The concept map is a useful way to derive and refine course content. Once analysts set global objectives that reflect the professional profiles, they can use the map to build a complete degree. 


\section{CURRICULUM DESIGN FRAMEWORKS}

Government and scientific organizations have proposed design frameworks for informatics, computing, and ICT curricula. Among these are the ICF-2000, commissioned by UNESCO; CC2001, jointly developed by IEEE and the ACM; and Career-Space, launched by the CSC. These initiatives differ in scope and coverage, but they are often complementary, which suggests the need for collaboration. For example, the Career-Space framework's demand focus could compensate for the lack of attention to demand aspects in CC2001. ${ }^{9}$

\section{Scope and purpose}

ICF-2000 is a curriculum framework rather than a precise set of guidelines. As such, it offers a broad and generic view, which means that each institution must design its own curriculum according to its set of institutional, cultural, and societal factors. In contrast, CC2001 focuses

\section{ICF-2000 offers a broad and generic view, which means that each institution must design its own curriculum.}

on computer science, offering a high-quality curriculum model derived from input involving many domain experts. Finally, Career-Space offers open-ended recommendations on a very global level about ICT curricula.

The final aim of each initiative is also different. ICF2000 aims for implementation in a certain context, while CC2001's detailed and precise curriculum guidelines restrict selection to certain model implementations. Finally, the Career-Space framework aims to fill the ICT skills gap as identified by the ICT industry.

\section{Methodology}

ICF-2000 follows a top-down methodology with a priori inclusion of all domains. It views informatics as broad and generic, to be analyzed or decomposed into several domains-computer science, computer engineering, software engineering, artificial intelligence, and so on. CC2001 follows a bottom-up approach with a step-by-step production of separate volumes, culminating in an overview. It synthesizes or composes computing from subdomains. The Career-Space framework views ICT as broad and generic, but basically merges traditional electrical engineering and informatics, adding business knowledge and behavioral skills.

\section{Orientation}

Both ICF-2000 and the Career-Space framework are demand-driven. The starting point in ICF-2000 is the workforce requirements, and the Career-Space framework considers a set of 13 ICT core generic skills profiles. In contrast, supply and academic requirements are the primary drivers for CC2001.

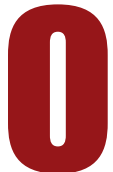

ur cross-analysis of ICT masters' programs in the EU with the CSC professional profiles shows that an alarming percentage of programs are not in line with the EHEA's employability aims. We hope that our findings serve as a reminder that curriculum design must consider the requirements of practitioners as well as theorists. If the curriculum fails to incorporate the needs and wants of ICT professionals, educators will not be fulfilling the demands of the ICT industry that eventually employs their graduates. The reformation projects are a solid step in the right direction. Curriculum designers must carry through that change mandate by implementing a top-down design approach and using scientific methodologies to satisfy the requirements of industry and society. C

\section{Acknowledgments}

This work was supported by the Spanish Ministry of Education and Science through studies and analysis subprogram EA2008-0042. We thank our project officers for their continued and enthusiastic assistance.

\section{References}

1. S. Schwarz and D.F. Westerheijden, "Accreditation in the Framework of Evaluation Activities: A Comparative Study in the European Higher Education Area," Accreditation and Evaluation in the European Higher Education Area, S. Schwarz and D.F. Westerheijden, eds., Higher Education Dynamics, vol. 5, Kluwer, 2004, pp. 1-41.

2. S.L. Toral et al., "An Electronic Engineering Curriculum Design Based on Concept-Mapping Techniques," 
Int'l J. Technology and Design Education, vol. 17, no. 3, 2007, pp. 341-356.

3. J. González and R. Wagenaar, eds., Final Report: Pilot Project Tuning Phase Carried out by over 100 Universities, University of Deusto, 2003.

4. D.S. Rychen and L.H. Salganik, eds., Key Competencies for a Successful Life and a Well-Functioning Society, Hogrefe and Huber, 2003.

5. F.E. Weinert, "Concept of Competence: A Conceptual Clarification," Defining and Selecting Key Competencies, D. Rychen and L. Salganik, eds., Hogrefe and Huber, 2001, pp. 45-65.

6. G. Salmon, "80:20 for E-Moderators," The Challenge of eCompetence in Academic Staff Development, I.M. Labhrainn et al., eds., NUI, 2006, pp. 145-155.

7. P.W.G. Bots and W.A.H. Thissen, "Negotiating Knowledge in Systems Engineering Curriculum Design: Shaping the Present while Struggling with the Past," IEEE Trans. Systems, Man and Cybernetics-Part C: Applications and Reviews, vol. 30, no. 2, 2000, pp. 197-203.

8. R.G. Carter, "Engineering Curriculum Design," IEE Proc., vol. 131, no. 9, 1984, pp. 678-683.

9. F. Mulder, K. Lemmen, and M. van Veen, "Variety in Views of University Curriculum," Informatics Curricula and Teaching Methods, L. Cassel and R. Reis, eds., Kluwer Academic Publishers, 2003, pp. 97-114.

Sergio Luis Toral is an associate professor in the Department of Electronic Engineering at the University of Seville. His research interests include e-learning, microprocessors, digital signal processors, real-time and distributed systems, open source software projects, and embedded operating systems. Toral received a PhD in electrical and electronic engineering from the University of Seville. He is a senior member of IEEE. Contact him at toral@esi.us.es.

Maria Rocio Martinez-Torres is an associate professor in the Department of Business Administration at the University of Seville. Her research interests include the evaluation of e-learning tools, knowledge management, concept mapping, and virtual communities of practice. Martinez-Torres received $a \mathrm{PhD}$ in business administration from the University of Seville. She is a member of the European Academy of Management and Business Economics. Contact her at rmtorres@us.es.

Federico Barrero is an associate professor in the Department of Electronic Engineering at the University of Seville. His research interests include embedded systems and electrical drives for industrial applications. Barrero received a $\mathrm{PhD}$ in electrical and electronic engineering from the University of Seville. He is a senior member of IEEE. Contact himatfbarrero@esi.us.es. 